\title{
Research on recycling channel selection of closed-loop supply chain considering consumer preference and fairness concern
}

\author{
Jiayi Sun ${ }^{1,2, *}$, Wei Chen ${ }^{1}$, Shuangfei Yang ${ }^{2}$, and Yingluo Yan ${ }^{2}$ \\ ${ }^{1}$ Harbin Engineering University, School of Economics and Management, Harbin, China \\ ${ }^{2}$ Harbin University of Science and Technology, School of Economics and Management, Harbin, \\ China
}

\begin{abstract}
This paper studied the influence of consumer preference and fairness concern on recycling and pricing decisions of closed-loop supply chain (CLSC) under different recycling channels. The results show that consumers' fairness concern cannot affect the manufacturer's decisions and profit; consumers' fairness concern is benefit to consumers, and it can improve the recycle rate. The channel selection strategy is depend on consumers' fairness attitude.
\end{abstract}

\section{Introduction}

Consumers' preferences for new and remanufactured products will influence the decisions of enterprises. Chiang et al. [1] studied this question earlier, then Xiong et al.[2] compared the advantages and disadvantages of manufacturers' remanufacturing and suppliers' remanufacturing under different preferences of consumers. Zhu et al.[3] studied the influence of consumer preference on consumers' purchasing decisions. Zhao et al.[4] discussed the influence of consumer preferences on pricing, servicing and recycling decisions of CLSC under different remanufacturing and patent licensing models.

With the rapid development of technology, the price information of products has become more transparent. For example, iFixit and Techinsights have analyzed the major components of iPhone and the overall BOM cost. The transparency of price information has aroused consumers' concern about the fairness of transaction. Cui et al.[5] proposed the coordination strategy under the condition that enterprises in supply chain have fairness concern behaviours. Zheng et al.[6] studied the coordination strategy of a three-level CLSC with retailers' fairness concern. Qin et al.[7] considered the effect of enterprises' fairness concern on supply chain decision-making under private cost information. Li et al.[8] discussed the impact of retailers' risk awareness and fairness concern on pricing decisions in a dual-channel supply chain. Shu et al.[9] studied the CLSC pricing decisions with fairness concern and competition among recyclers. The above researches focus on the enterprise fairness concern of supply chain or CLSC, but they ignore the psychology of consumers' fairness concern. Guo et al.[10] discussed the influence of consumers' aversion to unfair transactions on enterprises' quality and pricing decisions. Yi et al.[11] analysed the

\footnotetext{
* Corresponding author: sjy sjx@163.com
} 
influence of consumers' fairness concern on the choice of distribution channels. In conclusion, few literatures focus on the effect of consumers' preferences and fairness concern behaviours on recycling and pricing decisions in a CLSC, as well as the recycling channel selection strategy of the manufacture.

\section{Problem description and model assumptions}

The manufacturer is a leader in CLSC who decides to recycle used products for remanufacturing by herself or a third-party. The parties in the CLSC make a Stackelberg game with complete information. The associated notations are shown in Table 1.

Table 1. Summary of the notations.

\begin{tabular}{|c|c|}
\hline Notation & Meaning \\
\hline$p_{n}, p_{r}$ & Prices of new products and remanufactured products \\
\hline$w_{n}, w_{r}$ & $\begin{array}{l}\text { Wholesale prices of new products and remanufactured products, } \\
\qquad p_{n}>w_{n}, \quad p_{r}>w_{r}\end{array}$ \\
\hline$c$ & unit production cost of the new product \\
\hline$s$ & $\begin{array}{l}\text { relative to new product, the unit production cost of the } \\
\text { remanufactured product saved, } c>s\end{array}$ \\
\hline$\tau$ & remanufacturing rate, $0 \leq \tau \leq 1$ \\
\hline$A$ & $\begin{array}{c}\text { unit price of a returned product that the third party paid to the } \\
\text { customer }\end{array}$ \\
\hline$b$ & $\begin{array}{l}\text { unit price of a returned product that the manufacturer paid to the } \\
\text { third party, } A<b<s\end{array}$ \\
\hline$C(\tau)$ & $\begin{array}{c}\mathrm{C}(\tau)=k \tau^{2}, \text { the fixed investment of recycling used products, } k \text { is } \\
\text { the recovery cost coefficient, } k>0\end{array}$ \\
\hline$\theta$ & $\begin{array}{l}\text { consumers' willingness to pay for new products per unit, } \\
\qquad \theta \sim U(0,1)\end{array}$ \\
\hline$\delta$ & $\begin{array}{l}\text { consumers' acceptance of the remanufactured products, } \\
\qquad \delta \in(0,1)\end{array}$ \\
\hline$\lambda$ & the coefficient of consumers' fairness concern, $\lambda \in(0,1)$ \\
\hline
\end{tabular}

when consumer fairness concern is neutral, the demand functions are as follows:

$$
\begin{gathered}
q_{n}^{I}=1-\frac{p_{n}-p_{r}}{1-\delta} \\
q_{r}^{I}=\frac{\delta p_{n}-p_{r}}{\delta(1-\delta)}
\end{gathered}
$$

Considering that consumers are fairness concern, the demand functions are as follows:

$$
\begin{gathered}
q_{n}^{F}=1-\frac{(1+2 \lambda)\left(p_{n}-p_{r}\right)-\lambda\left(\omega_{n}-\omega_{r}\right)}{(1-\delta)(1+\lambda) \quad, \text { if } p_{n}<G} \\
q_{r}^{F}=\frac{(1+2 \lambda)\left(p_{n}-p_{r}\right)-\lambda\left(\omega_{n}-\omega_{r}\right)}{(1-\delta)(1+\lambda)}-\frac{(1+2 \lambda) p_{r}-\lambda \omega_{r}}{\delta(1+\lambda)} \text {, if } p_{n}<G
\end{gathered}
$$


where, $G=\frac{p_{r}}{\delta}+\frac{\lambda \omega_{n}}{(1+2 \lambda)}-\frac{\lambda \omega_{r}}{(1+2 \lambda) \delta}$.

\section{Model construction and solution}

When the third-party collecting the used products, the profit functions of manufacturer, retailer, and recycler are as follows:

$$
\begin{gathered}
\pi_{M}^{I D}\left(\omega_{n}, w_{r}\right)=\left(\omega_{n}-c\right) q_{n}+\left(\omega_{r}-c+s\right) q_{r}-b \tau\left(q_{n}+q_{r}\right) \\
\pi_{R}^{I D}\left(p_{n}, p_{r}\right)=\left(p_{n}-\omega_{n}\right) q_{n}+\left(p_{r}-\omega_{r}\right) q_{r} \\
\pi_{T}^{I D}(\tau)=(b-A) \tau\left(q_{n}+q_{r}\right)-k \tau^{2}
\end{gathered}
$$

Substituting equations (1-2) and equations (3-4) into equations (5-7) separately, the equilibrium results of considering consumer preferences and neutral fairness(the ID situation), as well as the equilibrium results of considering consumer preferences and fairness concern(the FD situation) can be obtained as shown in Table 2.

When the manufacturer collecting the used products, the profit functions of manufacturer and retailer are as follows:

$$
\begin{gathered}
\pi_{M}\left(\omega_{n}, \omega_{r}, \tau\right)=\left(\omega_{\mathrm{n}}-c\right) q_{n}+\left(\omega_{r}-c+s\right) q_{r}-b \tau\left(q_{n}+q_{r}\right)-k \tau^{2} \\
\pi_{R}\left(p_{n}, p_{r}\right)=\left(p_{n}-\omega_{n}\right) q_{n}+\left(p_{r}-\omega_{r}\right) q_{r}
\end{gathered}
$$

We can get the equilibrium results of considering consumer preferences and neutral fairness (the IE situation), as well as the equilibrium results of considering consumer preferences and fairness concern (the FE situation) in Table 2.

\section{Analysis of equilibrium results}

In order to ensure that the above equilibrium result is economically feasible, it is easy to know, $c<1, \delta>\frac{b(b-A)(1-s)+4 k(c-s)}{b(b-A)+4 k c}, c-s-c \delta<0, c-s<\delta<1-s, 8 \delta k-b^{2}<0$, $\frac{-b^{2}(s+1)}{b^{2}-8 k c-8 k}<\delta<\frac{b^{2}-2 k c+2 s k}{6 k}$.

Corollary $1 \frac{\partial \tau^{Y^{*}}}{\partial \delta}>0, \frac{\partial p_{n}^{Y^{*}}}{\partial \delta}>0, \frac{\partial q_{n}^{Y^{*}}}{\partial \delta}<0, \frac{\partial q_{r}^{Y^{*}}}{\partial \delta}>0, \frac{\partial \pi_{T}^{Y^{*}}}{\partial \delta}>0, \frac{\partial p_{r}^{I D^{*}}}{\partial \delta}>0$, when $\lambda>\lambda_{1}$, $\frac{\partial p_{r}^{F D^{*}}}{\partial \delta}>0 ;$ On the contrary, $\frac{\partial p_{r}^{F D^{*}}}{\partial \delta} \leq 0, \frac{\partial p_{r}^{I D^{*}}}{\partial \delta}>0, \frac{\partial p_{r}^{I D^{*}}}{\partial \delta}>0$.

where, $\lambda_{1}=\frac{k b(c+6 \delta-s)(b-A)-\left(b^{2}-A b\right)^{2}-12 k^{2} \delta^{2}}{k b(3 c+10 \delta-3 s)(b-A)+2\left(b^{2}-A b\right)^{2}+20 k^{2} \delta^{2}}, Y=\{I D, F D, I E, F E\}$.

Corollary $2 \frac{\partial p_{n}^{F D^{*}}}{\partial \lambda}<0, \frac{\partial p_{r}^{F D^{*}}}{\partial \lambda}<0, \frac{\partial \pi_{R}^{F D^{*}}}{\partial \lambda}<0, \frac{\partial p_{n}^{F E^{*}}}{\partial \lambda}>0, \frac{\partial p_{r}^{F E^{*}}}{\partial \lambda}>0$. 
Proposition $1 \tau^{I D^{*}}=\tau^{F D^{*}}, \omega_{n}^{I D^{*}}=\omega_{n}^{F D^{*}}, \omega_{r}^{I D^{*}}=\omega_{r}^{F D^{*}}, q_{n}^{I D^{*}}=q_{n}^{F D^{*}}, q_{r}^{I D^{*}}=q_{r}^{F D^{*}}$, $\pi_{M}^{I D^{*}}=\pi_{M}^{F D^{*}}, \pi_{T}^{I D^{*}}=\pi_{T}^{F D^{*}}, p_{n}^{I D^{*}}>p_{n}^{F D^{*}}, p_{r}^{I D^{*}}>p_{r}^{F D^{*}}, \pi_{R}^{I D^{*}}>\pi_{R}^{F D^{*}}$.

Proposition $2 \omega_{n}^{I E^{*}}=\omega_{n}^{F E^{*}}, \omega_{r}^{I E^{*}}=\omega_{r}^{F E^{*}}, q_{n}^{I E^{*}}=q_{n}^{F E^{*}}, \tau^{I E^{*}}=\tau^{F E^{*}}, q_{r}^{I E^{*}}=q_{r}^{F E^{*}}$, $\pi_{M}^{I E^{*}}=\pi_{M}^{F E^{*}}, p_{n}^{I E^{*}}<p_{n}^{F E^{*}}, p_{r}^{I E^{*}}<p_{r}^{F E^{*}}$.

Proposition 3 When $2 A / 3<b, \omega_{n}^{I E}>\omega_{n}^{I D}, \omega_{r}^{I E}>\omega_{r}^{I D}, q_{r}^{I E}<q_{r}^{I D} ; \omega_{n}^{I E} \leq \omega_{n}^{I D}, \omega_{r}^{I E} \leq \omega_{r}^{I D}$, $q_{r}^{I E} \geq q_{r}^{I D}, q_{n}^{I E}=q_{n}^{I D}, \tau^{I E}>\tau^{I D}$.

Proposition 4 When $2 A / 3<b, \omega_{n}^{F E}>\omega_{n}^{F D}, \omega_{r}^{F E}>\omega_{r}^{F D}, q_{r}^{F E}<q_{r}^{F D} ; \omega_{n}^{F E} \leq \omega_{n}^{F D}$, $\omega_{r}^{F E} \leq \omega_{r}^{F D}, q_{r}^{F E} \geq q_{r}^{F D}, q_{n}^{F E}=q_{n}^{F D}, \tau^{F E}>\tau^{F D}$.

Table 2. Equilibrium solution under the four models.

\begin{tabular}{|c|c|c|c|c|}
\hline & $I D$ & $F D$ & $I E$ & $F E$ \\
\hline$\omega_{n}^{*}$ & $\frac{D(\delta+s+1)-4 \delta}{2 D}$ & $\frac{D(\delta+s+1)-4 \delta k E}{2 D}$ & $\frac{H(\delta+s+1)-8}{2 H}$ & $\frac{H(\delta+s+1)-8 \delta k E}{2 H}$ \\
\hline$\omega_{r}^{*}$ & $\frac{(D-2 k E) \delta}{D}$ & $\frac{(D-2 k E) \delta}{D}$ & $\frac{(H-4 k E) \delta}{H}$ & $\frac{(H-4 k E) \delta}{H}$ \\
\hline$p_{n}^{*}$ & $\frac{D(\delta+s+3)-4 \delta}{4 D}$ & $\frac{D[(3 \lambda+1)(I+2)+2(\lambda+1)]}{4(2 \lambda+1) D}$ & $\frac{H(\delta+s+3)-8 \delta}{4 H}$ & $\frac{H[(3 \lambda+1)(I+2)+2(\lambda+1)]}{4(2 \lambda+1) H}$ \\
\hline$p_{r}^{*}$ & $\frac{(D-k E) \delta}{D}$ & $\frac{[D(2 \lambda+1)-k E(3 \lambda+1)] \delta}{(2 \lambda+1) D}$ & $\frac{(H-2 k E) \delta}{H}$ & $\frac{[H(2 \lambda+1)-2 k E(3 \lambda+1)]}{(2 \lambda+1) H}$ \\
\hline$\tau^{*}$ & $\frac{(b-A) E}{2 D}$ & $\frac{(b-A) E}{2 D}$ & $-\frac{b E}{H}$ & $-\frac{b E}{H}$ \\
\hline$q_{n}^{*}$ & $\frac{\delta+s-1}{4(\delta-1)}$ & $\frac{\delta+s-1}{4(\delta-1)}$ & $\frac{\delta+s-1}{4(\delta-1)}$ & $\frac{\delta+s-1}{4(\delta-1)}$ \\
\hline$q_{r}^{*}$ & $\frac{-D I+4(\delta-1) k l}{4(\delta-1) D}$ & $\frac{-D I+4(\delta-1) k E}{4(\delta-1) D}$ & $\frac{-H I+8(\delta-1) k E}{4(\delta-1) H}$ & $\frac{-H I+8(\delta-1) k E}{4(\delta-1) H}$ \\
\hline$\pi_{M}^{*}$ & $\frac{-D I^{2}+4(\delta-1) h}{8(\delta-1) D}$ & $\frac{-D I^{2}+4(\delta-1) k E}{8(\delta-1) D}$ & $\frac{-H I^{2}+8(\delta-1) k}{8(\delta-1) H}$ & $\frac{-H I^{2}+8(\delta-1) k E^{2}}{8(\delta-1) H}$ \\
\hline$\pi_{R}^{*}$ & $\frac{-D^{2} I^{2}+16 \delta(\delta}{16(\delta-1) L}$ & $\frac{(\lambda+1)\left[-D^{2} I^{2}+16 \delta(\delta-1\right.}{16(2 \lambda+1)(\delta-1) D}$ & $\frac{-H^{2} I^{2}+64 \delta(\delta}{16(\delta-1) H}$ & $\frac{(\lambda+1)\left[-H^{2} I^{2}+64 \delta(\delta-1\right.}{16(2 \lambda+1)(\delta-1) H}$ \\
\hline$\pi_{*}^{*}$ & $\frac{k(A-b)^{2} E^{2}}{4 D}$ & $\frac{k(A-b)^{2} E^{2}}{4 D}$ & & \\
\hline
\end{tabular}

where, $D=b^{2}-A b+4 \delta k, E=\delta+s-c, H=8 \delta k-b^{2}, I=\delta+s-1$

\section{Conclusion}

In the CLSC considering consumers' low-carbon preference and fairness concern, this paper discusses the impact of consumer fairness on the collecting and pricing decisions 
under different recycling channels. Comparing decision-makings and profits under four different models, the following conclusions are obtained: (1) When consumers are fairness neutrality, manufacturer-recycling situation is better to consumers and the retailer. (2) When consumers are fairness concern, third-party-recycling situation is better to consumers. (3) No matter who recycling used products, when consumers are fairness concern, under a certain condition, the sale price of products is lower and the recovery rate is higher.

\section{Reference}

1. Chiang W Y K, Chhajed D, Hess J D. Management Science, 49(1): 1-20, (2003).

2. Xiong Y, Zhao Q W, Zhou Y. International Journal of Production Economics, 176(1): 21-28, (2016).

3. Zhu X D, Yu L F. Applied Sciences, 8(7): 1207-1226, (2018).

4. Zhao J J, Wang C X, Xu L. Computers \& Industrial Engineering, 132: 59-73, (2019).

5. Cui T H, Raju J S, Zhang Z J. Management Science, 53(8): 1303-1314, (2007).

6. Zheng X X, Liu Z, Li K W, et al. International Journal of Production Economics, 212, 92-110, (2019).

7. Qin F,Mai F,Fry M J,et al.European Journal of Operational Research, 252(1):170-182, (2016).

8. Chun-Fa Li,Xue-Qing Guo,Dong-Lei Du. Journal of the Operations Research Society of China,2020(prepublish).

9. Shu Yadong,Dai Ying,Ma Zujun,Wang Xander.Mathematical Problems in Engineering,2020,(2020).

10. Guo X M,Jiang B J. Marketing Research, 53(5): 988-1000, (2016).

11. Yi Z L, Wang Y L.Production and Operations Management, 27 (6): 1148-1167, (2018).

12. Mark E, Ferguson L, Beril T. Production and Operations Management, 15(3): 351-368, (2006). 